\title{
Global variation in the long-term outcomes of ypT0 rectal cancers ${ }^{\text {th }}$
}

\author{
Laura Lorenzon ${ }^{\text {a, }}$, , Serge Evrard ${ }^{\text {b }}$, Geerard Beets ${ }^{\text {c}}$, Santiago Gonzalez-Moreno ${ }^{\text {d }}$, \\ Tibor Kovacs ${ }^{\mathrm{e}}$, Domenico D'Ugo a \\ Karol Polom ${ }^{\mathrm{f}, \mathrm{g}}$, on behalf CORSiCA Study - EYSAC.1 Study Group ${ }^{1}$ \\ ${ }^{a}$ General Surgery Unit, Fondazione Policlinico Universitario Agostino Gemelli IRCCS, Catholic University, Rome, Italy \\ ${ }^{\mathrm{b}}$ Institut Bergonié, Bordeaux, Cedex, France \\ ${ }^{c}$ Department of Surgery, Netherlands Cancer Institute, Amsterdam, Netherlands \\ d Surgical Oncology, MD Anderson Cancer Center, Madrid, Spain \\ e Breast Surgery Unit, Guy's Hospital, Guy's and St. Thomas' NHS Foundation Trust, London, UK \\ ${ }^{\mathrm{f}}$ Department of Surgical Oncology, Medical University of Gdansk, 80-211, Gdańsk, Poland \\ ${ }^{g}$ General Surgery and Surgical Oncology Department, University of Siena, 53100, Siena, Italy
}

\section{A R T I C L E I N F O}

\section{Article history:}

Received 30 April 2019

Received in revised form 12 September 2019

Accepted 10 October 2019

Available online $\mathrm{xxx}$

\section{Keywords:}

Rectal cancer

ypT0

Complete tumor response

Organ preservation

Local excision

\begin{abstract}
A B S T R A C T
Background: Colorectal cancer mortality presents world-wide variation. In rectal cancers presenting a complete/nearly-complete tumor response (ypT0/ypTis) following neoadjuvant treatment, the features correlated to nodal metastases and relapses still need to be defined.

Methods: An international cohort study enrolling ypT0/ypTis rectal cancers surgically treated from 2012 to 2017 was conducted. A propensity matching was used to balance nodal-positive and nodal-negative patients and statistical analyses were performed to investigate survivals, using a bootstrap model for internal validation. The features correlated with nodal metastasis were studied. Countries with participating centers were ranked using the World Bank (WBI), Human Development (HDI) and Global Gender Gap (GGG) indexes to compare survivals.

Results: 680 ypT0/ypTis from 52 European, Australian, Indian and American Institutions were analyzed. Mean follow-up was of 30.4 months. $96.5 \%$ were treated with total mesorectal excision, $7.2 \%$ were nodalpositive and $8.8 \%$ relapsed. Distal cancers (HR 0.71 95\%CI: 0.56-0.91) and nodal metastasis and nodal metastasis (HR 3.85 95\%CI:1.12-13.19) correlated with worse DFS, whereas a younger age was of borderline significance (HR 0.95 95\%CI:0.91-0.99). The bootstrap analysis validated the model on 5000 repetitions. A short-course radiotherapy (OR $0.1895 \% \mathrm{CI}: 0.09-0.37$ ) correlated with the occurrence of nodal metastasis. Those countries classified in the low/medium-WBI, medium-HDI and lower-GGG ranks documented worse DFS curves (respectively $\mathrm{p}<0.0001, \mathrm{p}<0.0001$ and $\mathrm{p} 0.0002$ ). However, the clinical stages were similar and patients from medium-HDI countries received more adjuvant chemotherapy than the others $(\mathrm{p}<0.0001)$.

Conclusion: Sub-groups at risk for relapses and nodal metastasis were identified. A global variation exists also when benchmarking a rectal cancer complete regression.
\end{abstract}

() 2019 Published by Elsevier Ltd.

\footnotetext{
This project was selected for presentation during the Scientific Symposium “Niall O'Higgins Award - Best Abstracts”, during the 38th Congress of the European Society of Surgical Oncology (ESSO 38), held in Budapest October 2018.

* Corresponding author. General Surgery Unit, Fondazione Policlinico Universitario Agostino Gemelli IRCCS, Catholic University, Largo Francesco Vito 1, 00168, Rome, Italy.

E-mail address: laura.lorenzon@policlinicogemelli.it (L. Lorenzon).

1 Annex 1. CORSiCA collaborator list including affiliations.
}

\section{Introduction}

Recent data from the Global Cancer Observatory (GLOBOCAN 2018), documented that colorectal cancer is still the third most frequent cancer and the second cause of cancer related mortality, however its incidence and mortality present a relevant world-wide variation $[1,2]$.

Rectal cancers account for about 30\% of colorectal cancers [3] and represent a field of relevant surgical, clinical and biological investigations. Over the last three decades the approach to rectal 
cancer radically changed: the improvements achieved lead to the introduction of total mesorectal excision (TME) and neoadjuvant (chemo)radiation treatments [4,5]. Nevertheless, the state-of-the art is continuously evolving as the effects of neoadjuvant treatments started to emerge in literature [6].

In particular, tumor down-staging following neoadjuvant treatment could result in a complete response, defined as clinical response (absence of residual primary tumor clinically detectable, cT0) or pathological response (absence of viable tumor cells within the rectal wall in the surgical specimen, ypT0) [7], occurring in about $10-20 \%$ of the patients who were treated with neoadjuvant therapy prior to surgery $[8,9]$.

In this subset of patients, the improved survival outcomes $[10,11]$ and the benefits of avoiding major surgical procedures, are encouraging a more conservative approach including watch and wait protocols $[12,13]$ or a local excision of the residual tumor scar [14].

Despite the achievement of a complete response could be acknowledged as a milestone, a number of issues still need to be addressed, in particular in relation to the surgical strategy, the identification of factors correlated to relapses and tumor regression, and the incidence and impact of a residual nodal disease.

A pilot multicenter investigation was recently conducted in this field investigating the pattern of survivals of rectal cancer patients presenting a complete or nearly complete tumor response after neoadjuvant therapy. Patients were treated using local excision or TME in Italy and Spain and results were highly promising, in particular in disclosing differences in survivals between patients assessed as nodal negative (ypN0) or presenting residual nodal metastases $(\mathrm{ypN}+)$ [6].

On the other hand, significant differences are emerging concerning survivals of rectal cancers in different countries, surprising also when comparing Norther European countries [15]. The geographic discrepancies concerning surgical quality and access to surgical care are currently a prioritizing issue, as widely declared by the Lancet Commission on Global Surgery [16]. On this extent, the National Institute for Health Research (NIHR) Global Health Research Unit on Global Surgery is in the process of establishing research hubs in low- and middle-income countries; a four-stage modified Delphy study identified three priority areas for future research, including the access to surgery, surgical oncology and peri-operative surgical care. With respect to the second domain, the aim was to define a resource-weighted quality assurance framework for cancer surgery; the research questions included, among the others, the identification of quality indicators and the role of multidisciplinary team meeting (MDT) in delivering cancer care. Accordingly, it was agreed that "a global observational cohort study was needed to benchmark care pathways and outcomes in lowincome against high-income countries. This study would capture data on patient pathways, including availability of diagnostic and therapeutic services, short-term surgical outcomes and longer-term cancerspecific outcomes". Colorectal cancer was assessed as a top priority along with breast and gastric cancers [17].

This study focused on COmplete pathological ReSponse rectal CAncer (CORSiCA) and aimed to investigate if nodal metastases independently affected prognosis and the clinical variables correlated with the occurrence of pathologic nodes. In addition, the global variations in the outcomes of rectal cancers presenting a complete pathological response were studied.

\section{Methods}

Design

This retrospective cohort study was promoted by the European
Society of Surgical Oncology (ESSO) Young Alumni Club (EYSAC). The project received approval by ESSO board and was registered on clinicaltrials.gov on November 2017 (ClinicalTrials.gov Identifier: NCT03351959). CORSiCA was publicized using ESSO network and social media and it was officially launched on December 1st. 2017 with a global call closing on March 2017. Actions were also taken by EYSAC steering committee members to spread the project; each center could participate a junior ( $<40$ years) member for data collection and a senior investigator for data validation [6]. No limit of enrollment was fixed for patients' registration, nor there was a minimum number of patients/center. The project was notified at the PI IRB institution (protocol n. 50973/17). The PI also standardized the core documentation, in order to have all sites working with the same version of the protocol and notified the centers with the project status updates using regular newsletters.

\section{Patients}

All patients with a rectal cancer surgically treated from 2012 to 2017, presenting a pathological surgical report consistent with ypT0 (absence of cancerous cells in the rectal wall) or ypTis (intramucosal carcinoma with no extension into the muscularis mucosae) following neoadjuvant treatment could be enrolled, independently from the neoadjuvant scheme, type of surgical resection or nodal status.

\section{Clinical and pathological records}

All the clinical and pathological records were de-identified by recruiting centers and pooled in a common database by the PI using a consecutive number. For the purpose of data collection, a database was designed adhering to the STROBE Statement [18]. The records included: demographics, tumor location and diameter, presence or not of large bowel obstruction, clinical staging (cTNM and clinical mesorectal fascia involvement - cMRF- defined as the presence or absence of tumor/nodal metastases involving mesorectal fascia on pre-treatment imaging scans) obtained using computer tomography (CT), magnetic resonance imaging (MRI) and/or endoscopic ultrasound (Endo-US). Also, the neoadjuvant protocols were collected, and patients classified into: short-course radiotherapy (SHORT RT) or a long-course chemo-radiotherapy (CHT-RT) sub-groups. The total dose of radiation was recorded (Gy), along with possible dose reductions or treatment interruptions. The radiological assessment following neoadjuvant treatment was recorded together with the interval time to surgery (measured in weeks). With respect to the surgical approaches, low-anterior resection, Miles resection and trans-anal TME were all categorized as TME procedures. On the other hand, patients undergoing a trans-anal endoscopic microsurgery resection, a trans-anal minimal invasive resection or a traditional trans-anal excision, were all grouped in the local excision group. The records included also the pathologic data (ypN stage, lymph-nodes harvested in the surgical specimen - LNH), the rate of adjuvant chemotherapy treatments and the long term oncological outcomes.

\section{Outcome measures}

The outcome measures included patients' survivals and residual nodal disease $(\mathrm{ypN}+)$. The follow-up was registered with the endpoints of overall survival (OS, any cause of death) and disease free survival (DFS, first recurrence after surgical resection). The relapses were differentiated in local relapses (rectal/anastomotic site), pelvic relapses (nodal) and relapses at distant sites (i.e. lung/liver). 


\section{Statistical analysis}

Continuous variables were analyzed using means and standard deviations (SD), tested for normal distribution using KolmogorovSmirnov normality test and compared accordingly. Categorical variables were analyzed using frequencies and percentage values and compared using Chi-square test. In order to control potential confounders that could affect the outcomes of interest, a propensity score matching [19] was used to generate two different treatment groups with balanced distribution of baseline features. Propensity scores resulted from logistic regression with dependent variable being the presence of nodal metastases. Covariates included age at diagnosis, gender and tumor location. The patients were matched one-to-two with the nearest-neighbor method using a caliper distance of $30 \%$ of the standard deviation of the logit of the estimated propensity score to ensure good matches. The balance between the two groups was assessed using the relative multivariate imbalance measure L1 as proposed by lacus, King and Porro [20-22].

Survival analyses were conducted using the Kaplan-Meier method with log-rank test and Cox regression analyses. The Hazard risk and its relative 95\% confidence interval (95\%CI) was estimated for each variable using the Cox proportional univariate model adopting the most suitable prognostic category as reference group. A multivariate Cox proportional hazard model was also developed using stepwise regression (forward selection). Enter limit and remove limit were $p=0.05$ and $p=0.10$, respectively, with significance defined at the $\mathrm{p}=0.05$ level. In order to avoid overfitting, a bootstrap method (resampling with replacement) was used for internal validation of the DFS univariate model. Ten independent procedures, each containing 1000 bootstrap samples, were performed. In order to further challenge the model, a larger procedure with 5000 repetitions was carried out.

To test if putative variables correlated with a ypN + outcome, a logistic regression was performed in the TME group including: gender, age, tumor location, cT, cN, cMRF stages and neoadjuvant schemes. The logistic regression was used since it has been documented an extremely valuable model in epidemiologic studies, allowing multiple variables to be analyzed in the same time. The odds ratio (OR) obtained expresses the probability of an event favorable to an outcome and the probability of an event against the same outcome; accordingly, a large OR means that the chance of a particular group is much greater than that of the reference group and vice-versa [23].

Finally, the geographic variation was investigated ranking countries with participating centers according to the World Bank Index (WBI) [24], the human development index (HDI: a United Nations' composite statistic including life expectancy, education, and income indices) [25] and the Global Gender Gap (GGG) Index provided by the World Economic Forum which measure progress towards gender parity across economic participation and opportunity, educational attainment, health and survival, and political empowerment [26]. Briefly, the countries were ranked into high income countries (HIC), upper middle income countries (UMIC) and lower middle income countries (LMIC) according to the WBI; very high/high HDI and medium HDI according to the United Nations' index and finally in the upper (1-50), middle (51-100) or lower (101-149) ranks of the GGG list report. The indexes were then compared for the differentiation of HIC-UMIC/very high-high HDI/ upper and middle GGG ranks vs LMIC/medium HDI/lower GGG ranks using the weighted kappa inter-agreement.

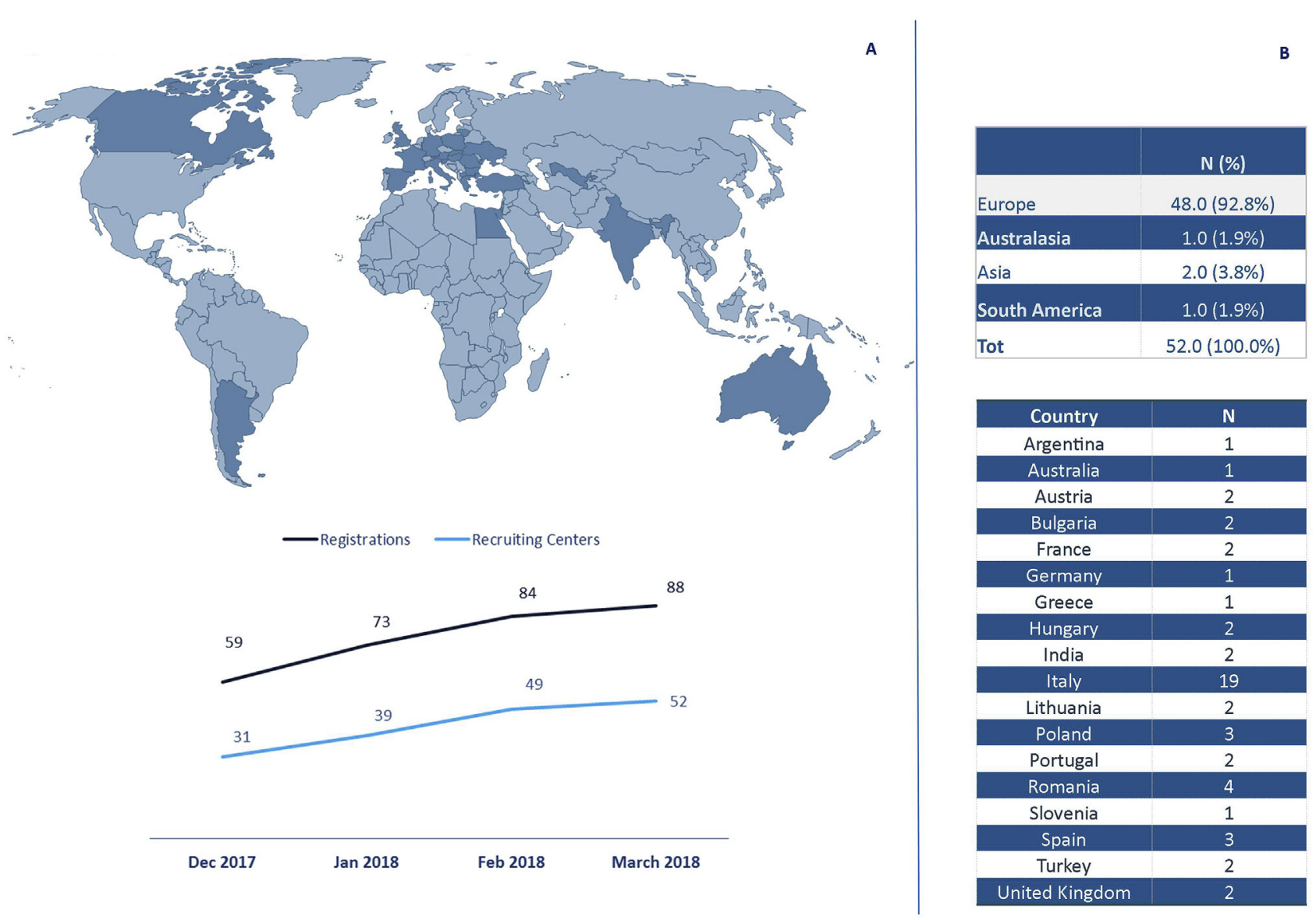

Fig. 1. CORSiCA Project. A. Registered Institutions map and trend over time. B. Number of participating Institutions according to different continents and countries. 
All statistical analyses were centralized by the PI, blinded to the recruiting centers and obtained using MedCalc (MariaKerke, Belgium) version 10.2.0.0 and SPSS (IBM, Armonk USA) version 21.0 software. All tests were performed two-tailed and a $\mathrm{p}$ value $<0.05$ was considered as statistically significant.

\section{Results}

Between December and March 2017, 88 Institutions from all over the world registered in the study, however exclusively 52 of them $(59.0 \%)$ performed as recruiting centers. About $93 \%$ of these 52 institutions were European, whereas non-European centers included Australia, India and Argentina, Fig. 1. Nine Institutions were also enrolled in the pilot investigation [6]. Mean age of the junior investigators was $32.9 \pm 4.2$ years whereas the seniors were about 15 years older (mean age $48.6 \pm 8.9$ years); the centers were mostly categorized as University hospitals (67.3\%), performing high volume colorectal surgery $(92.3 \%$ declared $>80$ colorectal resections/year) and with colorectal MDTs scheduled on a regular basis in $90.4 \%$ of the cases. Nine-hundred and seven ypT0/ypTis were registered, but patients with incomplete clinical records (date of surgery, age, clinical staging, neoadjuvant scheme, follow-up data, adjuvant therapy) and clinical stage IV were excluded, leaving 680 patients for data analysis. Overall, 77 patients, $11.3 \%$ of the case series, were enrolled in the same Institutions as the pilot investigation. Clinical and surgical data of the cohort are detailed in Table 1. Clinical staging was performed using MRI in $77.8 \%$ of the patients, CT scan in 97.3\%, and Endo-US in only $33.8 \%$ of the cases. The vast majority of the rectal cancers were clinically assessed as cT3/cT4 (84.6\%), cN+ (74.0\%), cMRF negative (74.0\%) before the neoadjuvant treatment.

As expected, a prevalence of males was reported (M/F 1.7) with a mean age of 60.0 years \pm 14.2 years. The majority of the patients presented a low rectal cancer (mean distance from the anal verge $5.8 \pm 3.2 \mathrm{~cm}$ ), and the neoadjuvant treatment consisted mostly of long-course CHT-RT (91.9\%). The tumors were mainly treated using TME, with only $3.5 \%$ of patients undergoing a local excision. Among TME patients, $7.1 \%$ were node positive. Some $85.4 \%$ of the SHORT RT sub-group was treated with delayed surgery at a mean interval of $12.9 \pm 11.9$ weeks. Mean follow up was $30.4 \pm 20.4$ months and $50.1 \%$ of the patients were subsequently treated with adjuvant chemotherapy following surgery. Sixty relapses were reported (8.8\%), thirty-eight at a distant site. The mean time to relapse was $20.9 \pm 13.3$ months.

\section{Nodal status and survival outcome}

The propensity method was conducted to match ypN + patients with ypNO patients from the TME group (ypN + experimental group $=1$, ypN0 control group $=0$; Matching Ratio 1:2), using the following co-variates: age, gender and tumor location. The analyses yielded 141 matched patients (1:2 ratio; $47 \mathrm{ypN}+$ and $94 \mathrm{ypN0}$ ). The L1 test measure was larger in the unmatched sample (0.764) than in the matched sample (0.553) indicating that the two groups were well balanced across all the variables considered. A lower rectal cancer (HR 0.71 95\%CI: 0.56-0.91) and ypN+ (HR 3.85 95\% CI:1.12-13.19) were variables correlated with worse DFS, whereas younger age was documented as a variable of borderline significance (HR 0.95 95\%Cl:0.91-0.99), Table 2. Although only the tumor location provided significant results in the multivariate model, all the three parameters were tested using a bootstrap.

A bootstrap method (resampling with replacement) was used for internal validation of the DFS univariate analysis. Ten independent procedures, each containing 5000 bootstrap samples, were performed and all of them validated the Cox proportional
Table 1

Clinical and pathological features of CORSiCA population.

\begin{tabular}{|c|c|c|}
\hline Sex & $\mathrm{N}$ & $\%$ \\
\hline $\mathbf{F}$ & 254.0 & 37.4 \\
\hline $\mathbf{M}$ & 425.0 & 62.5 \\
\hline Total & 680 & 100.0 \\
\hline $\mathbf{M} / \mathbf{F}$ & 1.7 & \\
\hline \multicolumn{3}{|l|}{ Age (years) } \\
\hline Mean (SD) & 60.0 & 14.2 \\
\hline \multicolumn{3}{|c|}{ Distance from the anal verge $(\mathrm{cm})$} \\
\hline Mean (SD) & 5.8 & 3.2 \\
\hline cT Stage & $\mathbf{N}$ & $\%$ \\
\hline cT1 & 4.0 & 0.6 \\
\hline cT2 & 97.0 & 14.3 \\
\hline cT3 & 512.0 & 75.3 \\
\hline cT4 & 67.0 & 9.9 \\
\hline Total & 680.0 & 100.0 \\
\hline cN Stage & $\mathbf{N}$ & $\%$ \\
\hline cNO & 177.0 & 26.0 \\
\hline $\mathbf{c N}+$ & 503.0 & 74.0 \\
\hline Total & 680.0 & 100.0 \\
\hline CMRF & $\mathbf{N}$ & $\%$ \\
\hline Negative & 503.0 & 74.0 \\
\hline Positive & 177.0 & 26.0 \\
\hline Total & 680.0 & 100.0 \\
\hline${ }^{\mathrm{b}}$ Neoadjuvant treatment & $\mathbf{N}$ & $\%$ \\
\hline CHT-RT & 625.0 & 91.9 \\
\hline SHORT-RT & 55.0 & 8.1 \\
\hline Total & 680.0 & 100.0 \\
\hline 'Surgical Treatment & $\mathbf{N}$ & $\%$ \\
\hline TME & 656.0 & 96.5 \\
\hline Local excision & 24.0 & 3.5 \\
\hline Total & 680.0 & 100.0 \\
\hline ypN Stage & $\mathbf{N}$ & $\%$ \\
\hline ypN + & 47 & 7.2 \\
\hline ypNo & 609 & 92.8 \\
\hline Total & 656.0 & 100.0 \\
\hline Adjuvant Chemotherapy & $\mathbf{N}$ & $\%$ \\
\hline Performed & 341 & 50.1 \\
\hline Not performed & 339 & 49.9 \\
\hline Total & 680.0 & 100.0 \\
\hline \multicolumn{3}{|l|}{ Follow up (months) } \\
\hline Mean (SD) & 30.4 & 20.4 \\
\hline Relapses & $\mathbf{N}$ & $\%$ \\
\hline Distant & 38.0 & 63.3 \\
\hline Local & 9.0 & 15.0 \\
\hline Distant + Local & 1.0 & 1.7 \\
\hline Pelvic & 7.0 & 11.7 \\
\hline Unknown & 5.0 & 8.3 \\
\hline Total & 60.0 & 100.0 \\
\hline
\end{tabular}

a cMRF: clinical mesorectal fascia involvement.

b Neo-adjuvant treatment - CHT-RT (long course chemoradiation) SHORT-RT (short course radiotherapy followed by immediate or delayed surgery).

c Surgical treatment: TME-total mesorectal excision.

hazard results, Table 3.

\section{Nodal metastases}

Table 4 shows results of logistic regression analysis. Also in this case the analysis was restricted to 656 TME patients. The logistic regression documented that a short-course radiotherapy (OR 0.18 95\%Cl:0.09-0.37) and younger age (OR 0.98 95\%Cl:0.96-0.99) correlated with the end-point of nodal metastasis, although age disclosed borderline values. Consistently with the effect of the radio-chemo-therapy, the long-course group had a significantly smaller nodal harvest (mean 12.3 vs 16.7, Kruskal-Wallis p-value 0.001), Suppl Fig. 1.

ypT0 in a global surgery frame

The patients from countries ranking in the LMIC, medium HDI 
Table 2

Cox proportional hazard model and nodal status: results from ypN $+v s$ ypNO PSM analysis.

\begin{tabular}{|c|c|c|c|c|c|}
\hline \multicolumn{6}{|l|}{ Endpoint $\mathrm{OS}^{\mathrm{a}}$} \\
\hline Variable & Comparison & HR & $95 \% \mathrm{CI}$ & p-value & \\
\hline Gender & $\mathrm{F}$ vs $\mathrm{M}$ & 0.78 & $0.20-3.15$ & 0.730 & \\
\hline Age & Continuous variable & 1.03 & $0.98-1.09$ & 0.222 & \\
\hline Tumor location & Continuous variable & 0.96 & $0.77-1.21$ & 0.744 & \\
\hline${ }^{\mathrm{C}}$ Neoadjuvant treatment & CHR-RT vs SHORT-RT & 23.91 & $\mathrm{NE}$ & - & \\
\hline ypN & ypN + vs ypN0 & 1.65 & $0.44-6.14$ & 0.458 & \\
\hline \multicolumn{6}{|l|}{ End-point DFS ${ }^{\mathrm{b}}$} \\
\hline & & \multicolumn{3}{|c|}{ Univariate model } & 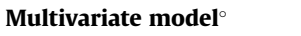 \\
\hline Variable & Comparison & HR & 95\%CI & p-value & \\
\hline Gender & $\mathrm{F}$ vs $\mathrm{M}$ & 0.39 & $0.08-1.82$ & 0.231 & \\
\hline Age & Continuous variable & 0.95 & $0.91-0.99$ & 0.008 & \\
\hline Tumor location & Continuous variable & 0.96 & $0.56-0.91$ & 0.006 & $0.71(0.56-0.91) \mathrm{p}=\mathbf{0 . 0 0 6}^{\circ}$ \\
\hline${ }^{\mathrm{c}}$ Neoadjuvant treatment & CHR-RT vs SHORT-RT & 0.88 & $0.11-7.09$ & 0.908 & \\
\hline ypN & ypN + vs ypN0 & 3.85 & $1.12-13.19$ & 0.032 & \\
\hline
\end{tabular}

Forward selection model

a OS: Overall Survival 9 events.

b DFS: Disease Free Survival 11 events

c Neo-adjuvant treatment - CHT-RT (long course chemoradiation) SHORT-RT (short course radiotherapy followed by immediate or delayed surgery).

Table 3

Bootstrap model-internal validation of DFS analysis in ypN + vs ypNO PSM sample.

\begin{tabular}{|c|c|c|c|c|c|}
\hline \multicolumn{6}{|l|}{ End-point DFS } \\
\hline \multirow[t]{2}{*}{ Variable } & \multirow[t]{2}{*}{ Comparison } & \multirow[t]{2}{*}{ HR } & \multirow[t]{2}{*}{ 95\%CI } & \multirow[t]{2}{*}{ p-value } & Bootstrap Results \\
\hline & & & & & $\mathrm{N}$ samples $=5000$ \\
\hline Age & Continuous variable & 0.95 & $0.91-0.99$ & 0.008 & Validated (10/10) \\
\hline Tumor location & Continuous variable & 0.71 & $0.56-0.91$ & 0.006 & Validated $(10 / 10)$ \\
\hline ypN & ypN + vs ypNO & 3.85 & $1.12-13.19$ & 0.032 & Validated $(10 / 10)$ \\
\hline
\end{tabular}

Table 4

Logistic regression with the end-point of $\mathrm{ypN}+$.

\begin{tabular}{lllll}
\hline Variable & Comparison & OR & $95 \% \mathrm{CI}$ & p-value \\
\hline Gender & F vs $\mathrm{M}$ & 0.87 & $0.47-1.63$ & 0.665 \\
Age & Continuous variable & 0.98 & $0.96-0.99$ & $\mathbf{0 . 0 4 0}$ \\
Tumor location & Continuous variable & 0.99 & $0.90-10.9$ & 0.796 \\
cT & $1+2$ vs 3 & 1.67 & $0.58-4.80$ & 0.343 \\
& $1+2$ vs 4 & 2.10 & $0.57-7.77$ & 0.266 \\
cN & cN + vs cN0 & 1.66 & $0.76-3.63$ & 0.204 \\
aMF & Positive vs Negative & 1.17 & $0.61-2.25$ & 0.630 \\
${ }^{b}$ Neoadjuvant treatment & CHR-RT vs SHORT-RT & 0.18 & $0.09-0.37$ & $<\mathbf{0 . 0 0 1}$ \\
\hline
\end{tabular}

a cMRF: clinical mesorectal fascia involvement.

b Neo-adjuvant treatment - CHT-RT (long course chemoradiation) SHORT-RT (short course radiotherapy followed by immediate or delayed surgery).

and those in the lower ranks of the GGG index documented worse DFS curves (respectively p value $<0.0001, p<0.0001$ and $p 0.0002$ ), Fig. 2. Interestingly, the clinical stages at presentation were reported similar in high and low income countries, p 0.183 (Table 5). Also, the patients from medium HDI countries received more adjuvant chemotherapy than the others, Table 5. Finally, the WBI and HDI indexes showed an optimal concordance in discriminating medium and lower-middle income ranks (weighted kappa 1.000), whereas both two indexes presented a moderate concordance vs GGG (weighted kappa 0.774), Suppl Table 1.

\section{Discussion}

The achievement of a complete pathologic response in the surgical specimen following neoadjuvant treatment is a benchmark of the progress made so far in rectal cancer treatment. Several manuscripts documented the benefits of complete response in terms of survival $[6,10]$ and clinical research is moving forward to explore the benefits of an organ preservation.
This study identified a group of "ugly features" in patients treated with TME; in particular, patients with distal and nodal positive tumors, reported a worse DFS; these results were strongly validated by the bootstrap model. Although in this study a younger age was documented an independent variable affecting prognosis of borderline significance, the literature reports that the incidence of colorectal cancer in individuals $<50$ years is escalating, and the tumor behavior in this sub-group has been described as particularly aggressive [27,28].

The presence of residual nodal disease in the in ypT0 sub-group herein reported was similar to past studies in this field, ranging between $6.7 \%$ and $8.7 \%[6,8]$. A recent analysis documented that the rate of ypT0-ypN + could be low to three percent if patients were clinically staged as nodal negative on pre-treatment MRI [29]. Past research reported also a strict correlation between the residual ypT and ypN following neoadjuvant treatment but no correlation was documented between ypN and tumor location [30]. In CAO/ARO/ AIO-04 trial, patients where noted to have no metastases below the tumor, and more nodal metastases in the peritumoral mesorectum than proximal to the tumor-site [31]. These results were not supported by the present findings where ypN + correlated with short-course radiotherapy but not with $\mathrm{cT} / \mathrm{cN}$ stages or tumor location; of note ypN + patients were registered in Australia, Germany, Greece, India, Italy, Poland, Portugal, Romania and Spain (data not shown).

These findings have significant implications when considering organ-preservation strategies. Nowadays, these approaches encompass a number of treatments ranging from endoscopical/ surgical local excisions to a full watch and wait approach [32,33]. Moreover, they imply the identification of a clinical complete response using radiological/radiomics criteria of tumor regression [34-37].

Undoubtedly, an organ preservation has the advantage of 
A

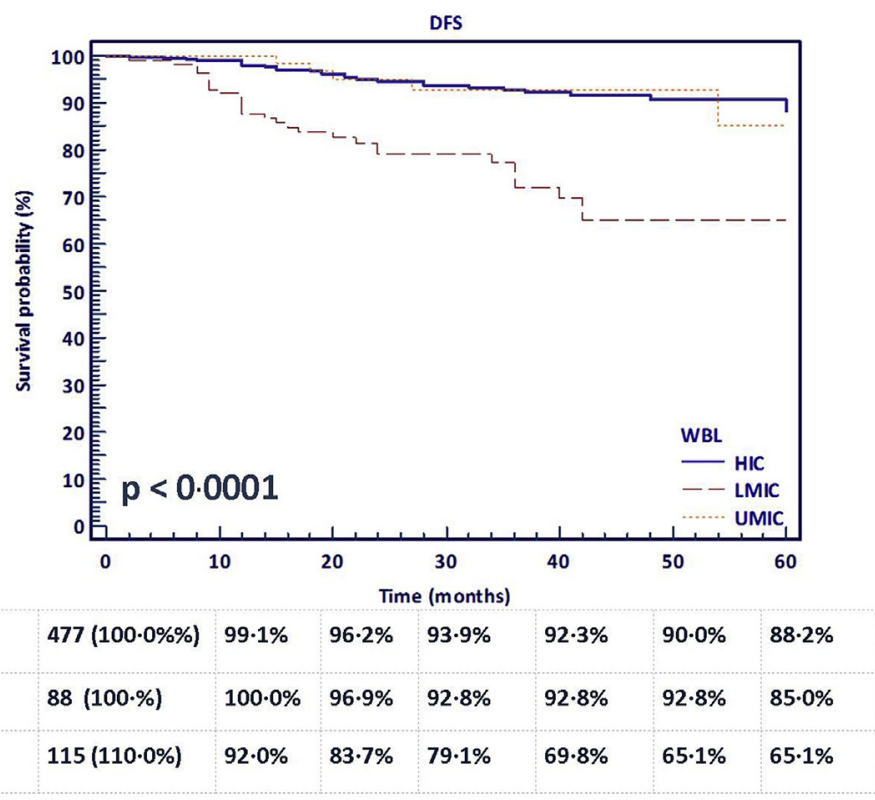

B

DFS

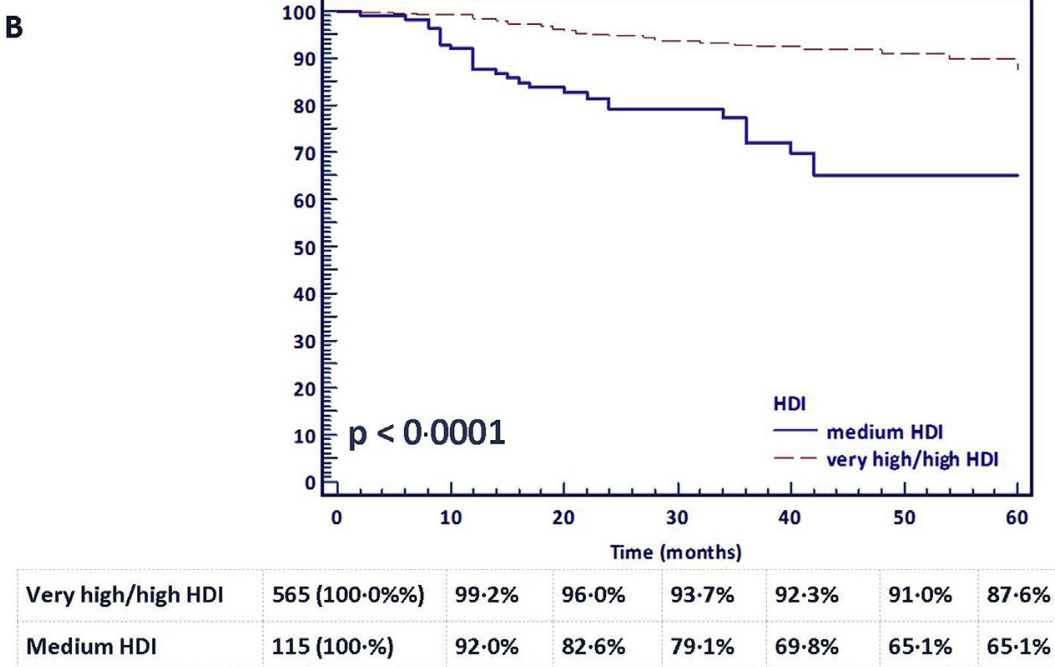

C

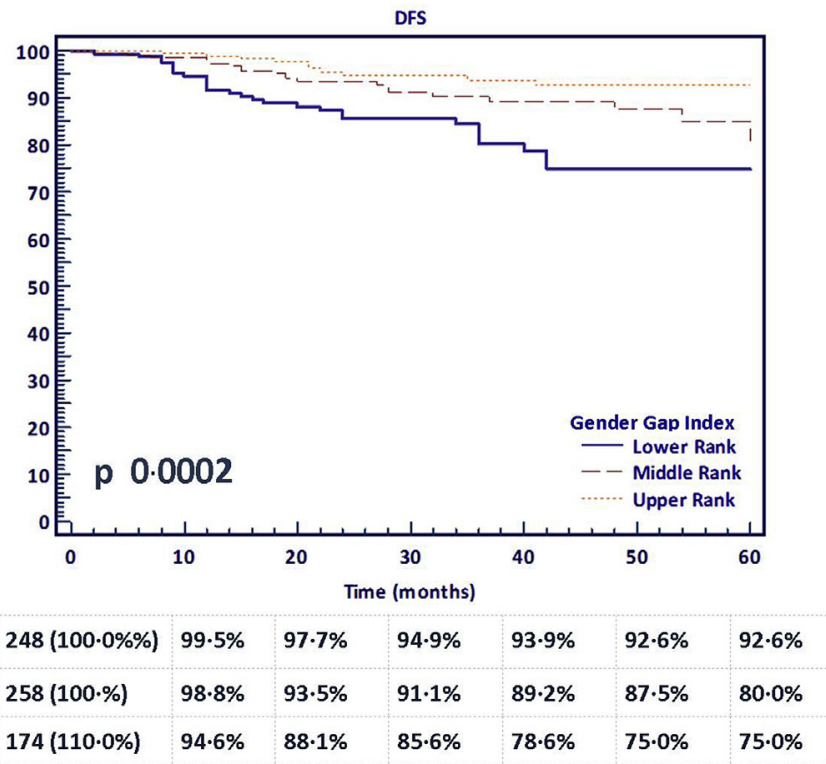

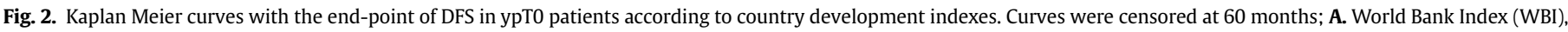
$\log$-rank test $\mathrm{p}<0.0001$; B. Human Development Index (HDI), log-rank test $\mathrm{p}<0.0001$; C. Gender Gap Index, log-rank test $\mathrm{p} 0.0002$.

Please cite this article as: Lorenzon L et al., Global variation in the long-term outcomes of ypT0 rectal cancers, European Journal of Surgical Oncology, https://doi.org/10.1016/j.ejso.2019.10.010 
Table 5

Clinical Stage and adjuvant treatments according to the economic and development indexes.

\begin{tabular}{|c|c|c|c|c|c|}
\hline \multirow[b]{2}{*}{ cStage } & \multicolumn{2}{|c|}{$\begin{array}{l}\text { high/very high } \\
\text { HDI }\end{array}$} & \multicolumn{2}{|c|}{ medium HDI } & \multirow[t]{2}{*}{$\mathrm{p}$ value } \\
\hline & $\mathrm{N}$ & $\%$ & $\%$ & $\%$ & \\
\hline cStage 1 & 39.0 & 6.9 & 3.0 & 2.6 & \\
\hline cStage 2 & 109.0 & 19.3 & 26.0 & 22.6 & 0.183 \\
\hline cStage 3 & 417.0 & 73.8 & 86.0 & 74.8 & \\
\hline Total & 565.0 & 100.0 & 115.0 & 100.0 & \\
\hline Adjuvant Chemotherapy & $\mathbf{N}$ & $\%$ & $\mathbf{N}$ & $\%$ & \\
\hline Performed & 235.0 & 41.6 & 106.0 & 92.2 & $<0.0001$ \\
\hline Not performed & 330.0 & 58.4 & 9.0 & 7.8 & \\
\hline Total & 565.0 & 100.0 & 115.0 & 100.0 & \\
\hline
\end{tabular}

ensuring a better quality of life, although the follow-up schemes are more intensive and the local tumor regrowth has been acknowledged as a critical issue $[13,38,39]$. Recent results from the InterCoRe consortium investigated the factors affecting local regrowth in 600 patients managed with watch and wait, using an individual participant data meta-analysis. The cumulative incidence of local regrowth was of $21.4 \%$ and correlated with cT stage, reporting, however, high levels of heterogeneity between studies [40]. Similar results were also obtained in the OnCoRe Project, although local regrowth rates were higher (34\%) [41].

Possibly because of the difficulties in defining a clinical response, but also due to the limitations of the researches published so far, the organ preservation strategy is not currently recommended in routinely practice out of clinical studies. According to the most recent ESMO guidelines this approach could be considered when a clinical response is achieved in high-risk/fragile patients; however, "a small increased oncological risk of pelvic and metastatic disease exist, although the prognosis is excellent even without surgery" [42]. The results from large studies, such as the IWWD registry $[43,44]$ or the STAR-TREC trial [45], will provide more information and will help in selecting eligible patients.

A particular aspect of this large data-set is the involvement of several countries from four continents, aiming to frame the results of complete response in a global surgery context. As previously reported, surgery is the gold standard treatment of solid tumors, but currently less than one quarter of patients currently receive a safe, affordable and timely procedures, since surgical care in lowincome countries is largely neglected [16]. However, this issue is affecting also northern Europe, since survival from colorectal cancer in England and Denmark was recently reported lower than in Norway and Sweden [15]. Remarkably, this study reported optimal survivals in high-income ranking countries according to three different, but concordant, indexes. A significant negative correlation between relapses and low-income countries was documented on Kaplan Meier analyses, despite all patients performed neoadjuvant therapy and all of them received a surgical treatment (at least with a local excision).

If a late presentation could explain the variances in disease free survivals, CORSiCA study disclosed that no particular difference was noted concerning clinical stages at presentation among different countries. Still, survival outcomes could be explained on the basis of differences in the tumor biology, but patients were registered in four continents in a precise time frame. This study highlighted also that medium-HDI countries perform more adjuvant chemotherapy comparing high/very high-HDI countries, consistently with the multimodal management. Despite almost the totality of the Institutions participating in this project, self-declared to perform $>80$ colorectal resections/year and to discuss patients in MDTs on a regular basis, it should be acknowledged that volume is not the only key indicator of quality and the rise in surgical standards as well as the achievement of a multimodal treatment in a process of continuous auditing were the elements that changed the state of the art in rectal cancer management $[46,47]$.

Recently, the HDI was used to compare countries on the basis of the surgical curriculum they offered. It was documented that the length of training and the availability of domestic surgical oncology fellowships had a positive correlation with HDI ranks [48]. On this basis, the leaders of the Society of Surgical Oncology and European Society of Surgical Oncology developed a global curriculum to incorporate the domains considered to be essential in surgical oncology [49].

Limitations of this study include the observational design and the impossibility of conducting molecular analyses to investigate features correlated with residual nodal disease. However, the large sample of patients from all over the world allowed to obtain significant clinical data, as recently achieved in other large international audits [50,51].

Finally, the schemes of neoadjuvant treatment for rectal cancer varies considerably across different countries [52]. A recent study in this field demonstrated that between 2007 and 2014 the use of SHORT RT ranged from $5 \%$ in Belgium to $75 \%$ in the Netherlands, whereas CHT-RT was used in the $87 \%$ of patients in Belgium and in the $15 \%$ of stage II-III rectal cancers in the Netherlands [52]. However, the vast majority of neoadjuvant protocols are based on CHTRT schemes, consistently with our results, although they may present variation in the rate of ypT0 produced. These results are in relation to the radiation dose, the chemotherapy drugs and the interval to surgery [53]; all these features may vary also in the same Institution through the years. During the study period, some 17687 rectal cancers where surgically treated across participating Institutions (mean 340.1 \pm 338.8 patients, ranging 52-1760 surgical procedures/Institution). On this basis, the rate of complete responses reported in this study is consistent with the overall population included. Of note, even if patients were treated by different means and modalities, the entire project focused on the ultimate effect of the neoadjuvant treatment: the occurrence of a complete rectal cancer regression, thus the cases selected across sites where homogeneous for this feature.

The findings reported with CORSiCA project have important oncological and oncopolicy implications. This project identified a particular sub-group of responders patients at risk for relapses, including patients with distal tumors and those reporting the persistence of nodal disease. In particular and with respect of the nodal persistence, a short course radiotherapy was documented in relation with $\mathrm{ypN}+$. All these features should be taken into account when considering a more conservative approach.

Finally, since the clinical stages and rates of adjuvant treatment were comparable, two possible reasons could explain the geographic discrepancies, one and highly speculative being the difference in the tumor biology that was not here investigated. The second reason could be a possible shortfall in the provision of surgical oncology standards. Raising the standards in surgical oncology quality should improve the long-term outcomes globally, also when the effect of the multimodal treatment of rectal cancer achieve a complete tumor regression. However, the addition of molecular features would help in better understanding the international variation and possible inequalities.

\section{Funding}

None. 


\section{Declaration of competing interest}

None of the authors has any potential financial conflict of interest related to this manuscript.

\section{Appendix A. Supplementary data}

Supplementary data to this article can be found online at https://doi.org/10.1016/j.ejso.2019.10.010.

\section{References}

[1] https://www.uicc.org/new-global-cancer-data-globocan-2018.

[2] http://gco.iarc.fr/today/home.

[3] Siegel RL, Miller KD, Jemal A. Cancer statistics. CA Cancer J Clin 2016:66:7-30.

[4] van Gijn W, Marijnen CA, Nagtegaal ID, Kranenbarg EM, Putter H, Wiggers T, et al. Preoperative radiotherapy combined with total mesorectal excision for resectable rectal cancer: 12-year follow-up of the multicentre, randomised controlled TME trial. Lancet Oncol 2011;12:575-82.

[5] Heald RJ, Ryall RD. Recurrence and survival after total mesorectal excision for rectal cancer. Lancet 1986;1:1479-82.

[6] Lorenzon L, Parini D, Rega D, Mellano A, Vigorita V, Biondi A, et al. Long-term outcomes in ypT0 rectal cancers: an international multi-centric investigation on behalf of Italian Society of Surgical Oncology Young Board (YSICO). Eur J Surg Oncol 2017;43:1472-80.

[7] Habr-Gama A, Perez RO, Nadalin W, Sabbaga J, Ribeiro Jr U, Silva e Sousa Jr AH, et al. Operative versus nonoperative treatment for stage 0 distal rectal cancer following chemoradiation therapy: long-term results. Ann Surg 2004;240: $711-7$.

[8] Al-Sukhni E, Attwood K, Mattson DM, Gabriel E, Nurkin SJ. Predictors of pathologic complete response following neoadjuvant chemoradiotherapy for rectal cancer. Ann Surg Oncol 2016;23:1177-86.

[9] 2017 European Society of Coloproctology (ESCP) collaborating group. Evaluating the incidence of pathological complete response in current international rectal cancer practice: the barriers to widespread safe deferral of surgery. Colorectal Dis 2018;20(Suppl 6):58-68.

[10] Maas M, Nelemans PJ, Valentini V, Das P, Rödel C, Kuo LJ, et al. Long-term outcome in patients with a pathological complete response after chemoradiation for rectal cancer: a pooled analysis of individual patient data. Lancet Oncol 2010;11:835-44.

[11] Martin ST, Heneghan HM, Winter DC. Systematic review and meta-analysis of outcomes following pathological complete response to neoadjuvant chemoradiotherapy for rectal cancer. Br J Surg 2012;99:918-28.

[12] Habr-Gama A, Gama-Rodrigues J, Sao Juliao GP, Proscurshim I, Sabbagh C, Lynn PB, et al. Local recurrence after complete clinical response and watch and wait in rectal cancer after neoadjuvant chemoradiation: impact of salvage therapy on local disease control. Int J Radiat Oncol Biol Phys 2014;88:822-8.

[13] Kong JC, Guerra GR, Warrier SK, Ramsay RG, Heriot AG. Outcome and salvage surgery following "watch and wait" for rectal cancer after neoadjuvant therapy: a systematic review. Dis Colon Rectum 2017;60:335-45.

[14] Capirci C, Valentini V, Cionini L, De Paoli A, Rodel C, Glynne-Jones R, et al. Prognostic value of pathologic complete response after neoadjuvant therapy in locally advanced rectal cancer: long-term analysis of 566 ypCR patients. Int J Radiat Oncol Biol Phys 2008;72:99-107.

[15] Benitez Majano S, Di Girolamo C, Rachet B, Maringe C, Guren MG, Glimelius B, et al. Surgical treatment and survival from colorectal cancer in Denmark, England, Norway, and Sweden: a population-based study. Lancet Oncol 2019;20:74-87.

[16] Meara JG, Leather AJ, Hagander L, Alkire BC, Alonso N, Ameh EA, et al. Global Surgery 2030: evidence and solutions for achieving health, welfare, and economic development. Lancet 2015;386:569-624.

[17] National Institute for Health Research Global Health Research Unit on Global Surgery. Prioritizing research for patients requiring surgery in low- and middle-income countries. Br J Surg 2019;106:e113-20.

[18] von Elm E, Altman DG, Egger M, Pocock SJ, Gøtzsche PC, Vandenbroucke JP, et al. The strengthening the reporting of observational studies in epidemiology (STROBE) statement: guidelines for reporting observational studies. Lancet 2007;370:1453-7.

[19] Austin PC. Comparing paired vs non-paired statistical methods of analyses when making inference about absolute risk reduction in propensity score matched samples. Stat Med 2011;30:1292-301.

[20] Hansen BB, Bowers J. Covariate balance in simple, stratified and clustered comparative studies. Stat Sci 2008;23:219-36.

[21] Iacus SM, King G, Porro G. CEM: coarsened exact matching software. J Stat Softw 2009;30:1-27. https://doi.org/10.18637/jss.v030.i09.

[22] Iacus SM, King G, Porro G. Causal Inference without Balance checking: coarsened exact matching. Political Anal 2011;20:1-24.

[23] Sperandei S. Understanding logistic regression analysis. Biochem Med 2014;24:12-8.

[24] https://databank.worldbank.org/data/source/world-development-indicators.

[25] http://hdr.undp.org/en/content/human-development-index-hdi.
[26] https://www.weforum.org/reports/the-global-gender-gap-report-2018.

27] Pilozzi E, Lorenzon L, Lo Baido S, Ferri M, Duranti E, Fochetti F, et al. Left-sided early onset colorectal carcinomas: a sporadic neoplasm with aggressive behavior. Am J Surg 2017;214:421-7.

[28] Weinberg BA, Marshall JL. Colon cancer in young adults: trends and their implications. Curr Oncol Rep 2019;21:3.

[29] Wan J, Liu K, Zhu J, Li G, Zhang Z. Implications for selecting local excision in locally advanced rectal cancer after preoperative chemoradiation. Oncotarget 2015;6:11714-22.

[30] Bosch SL, Vermeer TA, West NP, Swellengrebel HA, Marijnen CA, Cats A, et al. Clinicopathological characteristics predict lymph node metastases in ypT0-2 rectal cancer after chemoradiotherapy. Histopathology 2016;69:839-48.

[31] Sprenger T, Rothe H, Becker H, Beissbarth T, Homayounfar K, Gauss K, et al Lymph node metastases in rectal cancer after preoperative radiochemotherapy: impact of intramesorectal distribution and residual micrometastatic involvement. Am J Surg Pathol 2013;37:1283-9.

[32] Stijns RCH, Tromp MR, Hugen N, de Wilt JHW. Advances in organ preserving strategies in rectal cancer patients. Eur J Surg Oncol 2018;44:209-19.

[33] Barina A, De Paoli A, Delrio P, Guerrieri M, Muratore A, Bianco F, et al. Rectal sparing approach after preoperative radio- and/or chemotherapy (RESARCH) in patients with rectal cancer: a multicentre observational study. Tech Coloproctol 2017;21:633-40.

[34] Habr-Gama A, Perez RO, Wynn G, Marks J, Kessler H, Gama-Rodrigues J. Complete clinical response after neoadjuvant chemoradiation therapy for distal rectal cancer: characterization of clinical and endoscopic findings for standardization. Dis Colon Rectum 2010;53:1692-8.

[35] Siddiqui MR, Gormly KL, Bhoday J, Balyansikova S, Battersby NJ, Chand M, et al. Interobserver agreement of radiologists assessing the response of recta cancers to preoperative chemoradiation using the MRI tumour regression grading (mrTRG). Clin Radiol 2016:71:854-62.

[36] Battersby NJ, Dattani M, Rao S, Cunningham D, Tait D, Adams R, et al. A rectal cancer feasibility study with an embedded phase III trial design assessing magnetic resonance tumour regression grade (mrTRG) as a novel biomarke to stratify management by good and poor response to chemoradiotherapy (TRIGGER): study protocol for a randomised controlled trial. Trials 2017; 18 : 394.

[37] Cusumano D, Dinapoli N, Boldrini L, Chiloiro G, Gatta R, Masciocchi C, et al. Fractal-based radiomic approach to predict complete pathological response after chemo-radiotherapy in rectal cancer. Radiol Med 2018;123:286-95.

[38] Hupkens BJP, Martens MH, Stoot JH, Berbee M, Melenhorst J, Beets-Tan RG, et al. Quality of life in rectal cancer patients after chemoradiation: watch-andwait policy versus standard resection - a matched-controlled study. Dis Colon Rectum 2017;60:1032-40.

[39] Dossa F, Chesney TR, Acuna SA, Baxter NN. A watch-and-wait approach for locally advanced rectal cancer after a clinical complete response following neoadjuvant chemoradiation: a systematic review and meta-analysis. Lancet Gastroenterol Hepatol 2017;2:501-13.

[40] Chadi SA, Malcomson L, Ensor J, Riley RD, Vaccaro CA, Rossi GL, et al. Factors affecting local regrowth after watch and wait for patients with a clinical complete response following chemoradiotherapy in rectal cancer (InterCoRe consortium): an individual participant data meta-analysis. Lancet Gastroenterol Hepatol 2018:3:825-36.

[41] Renehan AG, Malcomson L, Emsley R, Gollins S, Maw A, Myint AS, et al Watch-and-wait approach versus surgical resection after chemoradiotherapy for patients with rectal cancer (the OnCoRe project): a propensity-score matched cohort analysis. Lancet Oncol 2016;17:174-83.

[42] Glynne-Jones R, Wyrwicz L, Tiret E, Brown G, Rodel C, Cervantes A, et al. Recta cancer: ESMO clinical practice guidelines for diagnosis, treatment and followup. Ann Oncol 2017;28(suppl_4). iv22-iv40.

[43] Van de Valk M. The International Watch and Wait database (IWWD) for rectal cancer: an update. J Clin Oncol 2017;35(Suppl 4S). abstr 521.

[44] van der Valk MJM, Hilling DE, Bastiaannet E, Meershoek-Klein Kranenbarg E, Beets GL, Figueiredo NL, et al. Long-term outcomes of clinical complete responders after neoadjuvant treatment for rectal cancer in the Internationa Watch \& Wait Database (IWWD): an international multicentre registry study. Lancet 2018;391:2537-45.

[45] Rombouts AJM, Al-Najami I, Abbott NL, Appelt A, Baatrup G, Bach S, et al. Can we save the rectum by watchful waiting or transanal microsurgery following (chemo) radiotherapy versus Total mesorectal excision for early rectal cance (STAR-TREC study)?: protocol for a multicentre, randomised feasibility study BMJ Open 2017;7:e019474.

[46] den Dulk M, Krijnen P, Marijnen CA, Rutten HJ, van de Poll-Franse LV, Putter $\mathrm{H}$, et al. Improved overall survival for patients with rectal cancer since 1990: the effects of TME surgery and pre-operative radiotherapy. Eur J Cancer $2008 ; 44: 1710-6$.

[47] Kodeda K, Johansson R, Zar N, Birgisson H, Dahlberg M, Skullman S, et al. Time trends, improvements and national auditing of rectal cancer management over an 18-year period. Colorectal Dis 2015;17:0168-79.

[48] Are C, Caniglia A, Malik M, Cummings C, Lecoq C, Berman R, et al. Variations in training of surgical oncologists: proposal for a global curriculum. Eur J Surg Oncol 2016;42:767-78.

[49] Are C, Berman RS, Wyld L, Cummings C, Lecoq C, Audisio RA. Global Curriculum in surgical oncology. Ann Surg Oncol 2016;23:1782-95.

[50] GlobalSurg Collaborative. Surgical site infection after gastrointestinal surgery in high-income, middle-income, and low-income countries: a prospective. 
international, multicentre cohort study. Lancet Infect Dis 2018;18:516-25.

[51] GlobalSurg Collaborative. Pooled analysis of WHO Surgical Safety Checklist use and mortality after emergency laparotomy. Br J Surg 2019;106: e103-12.

[52] Babaei M, Jansen L, Balavarca Y, Sjövall A, Bos A, van de Velde T, et al. Neoadjuvant therapy in rectal cancer patients with clinical stage II to III across
European countries: variations and outcomes. Clin Colorectal Cancer 2018;17: e129-42.

[53] Ryan E, O'Sullivan DP, Kelly ME, Syed AZ, Neary PC, O'Connell PR, et al. Metaanalysis of the effect of extending the interval after long-course chemoradiotherapy before surgery in locally advanced rectal cancer. Br J Surg 2019;106:1298-310.

Please cite this article as: Lorenzon L et al., Global variation in the long-term outcomes of ypT0 rectal cancers, European Journal of Surgical Oncology, https://doi.org/10.1016/j.ejso.2019.10.010 